\title{
A High Precision Load Cell Mass Comparator
}

\author{
Randall M. Schoonover \\ Center for Absolute Physical Quantities, National Bureau of Standards, Washington, DC 20234 \\ June 13, 1979

\begin{abstract}
Described here is a simple mechanical method used to fabricate a high precision mass comparator using a bonded strain gage load cell. Results indicate that a standard deviation of less than $0.0003 \%$ is readily attainable, and the device works well for objects normally considered too unwieldy for large high-precision balances.

Key words: Constant loading; force; high precision; high-precision weighing; load cell; mass; mass comparator; mass difference; strain-gage; substitution weighing; weighing; weights.
\end{abstract}

\section{Introduction}

It is generally accepted in metrology that the most accurate assignment of mass to an object is accomplished by a difference measurement between a standard of mass and the object of interest. Usually this difference measurement, or comparison, as it is called, is accomplished with either a lever scale or a balance, depending on the magnitude of mass involved.

In recent years another type of mass comparator, based on nearly constant loading of a bonded strain gage load cell, was constructed by Gilmore Industries ${ }^{1}$, and tested by the National Bureau of Standards. The concept $[1]^{2}$ is to maintain the cell at nearly constant load during the periods it would normally be unloaded. This is accomplished by electronic servo-control of a hydraulic force generated by a piston and cylinder and applied to the cell, resulting in a precision not ordinarily attained with a load cell.

The mechanism presented here is a simple mechanical device designed to provide the nearly constant load force required for accurate results.

\subsection{Background}

In general, the widest application for load cells is as direct-reading force measuring instruments. Initially the cell is calibrated by the application of known forces and the output is noted. Since the cells have electronic outputs, the cell and associated electronics are often calibrated as a system and in most instances have an uncertainty of at least 0.05 percent when used to measure an unknown force. When the load cell is used simply as a mass comparator (i.e., force comparator) according to the method presented here, the uncertainty is reduced to about 0.005 percent.

\footnotetext{
${ }^{1}$ Button, Donald M., The development of a mobile mass comparator, paper presented at the 20th annual ISA Conference October 4-7, 1965 (not published).

${ }^{2}$ Figures in brackets indicate the literature references at the end of this paper.
}

In the standards laboratory it is often necessary to assign mass values at the 0.0001 percent level of uncertainty to objects of nominally $225 \mathrm{~kg}(500 \mathrm{lb})$ and larger. Obviously a standard deviation of at least $1 \mathrm{ppm}$ is desirable as imprecision degrades the transfer of mass information from the standard weight to the unknown object. This capability is far beyond what load cells, as ordinarily used, can achieve. However, with constant loading of the cell during the exchange of objects being compared (standard weights for example) standard deviations of less than the 0.0003 percent level are attainable.

Fortunately, the equipment required for constant loading is mostly "off-the-shelf" and only simple mechanical structures have to be fabricated.

\section{The Load Cell Comparator}

Maintaining the loading on the cell during the interchange of objects being compared eliminates much of the hysteresis and "creep." The arrangement described here uses the force exerted by a coil spring to maintain about 90 percent of the load during the interchange of objects. Essentially, this is accomplished by suspending the cell through a coil spring, allowing the load to compress the spring and then adjusting the surrounding frame to prevent removal of more than 10 percent of the spring force. After initial adjustment, nominally equal masses can be compared. The schematic, figure 1 , will aid the reader in following a more detailed discussion.

The lifting eye carries the frame and is connected to a hydraulic lifter that can be suspended from a building structural member, A-frame, etc. The frame is rigid and receives the forces exerted by the objects to be compared.

A coil spring, with a predetermined spring constant and length, rests upon the bed plate and is centered about a passageway. On the upper end of the spring rests a floating plate with its passageway likewise centered. Horizontal 


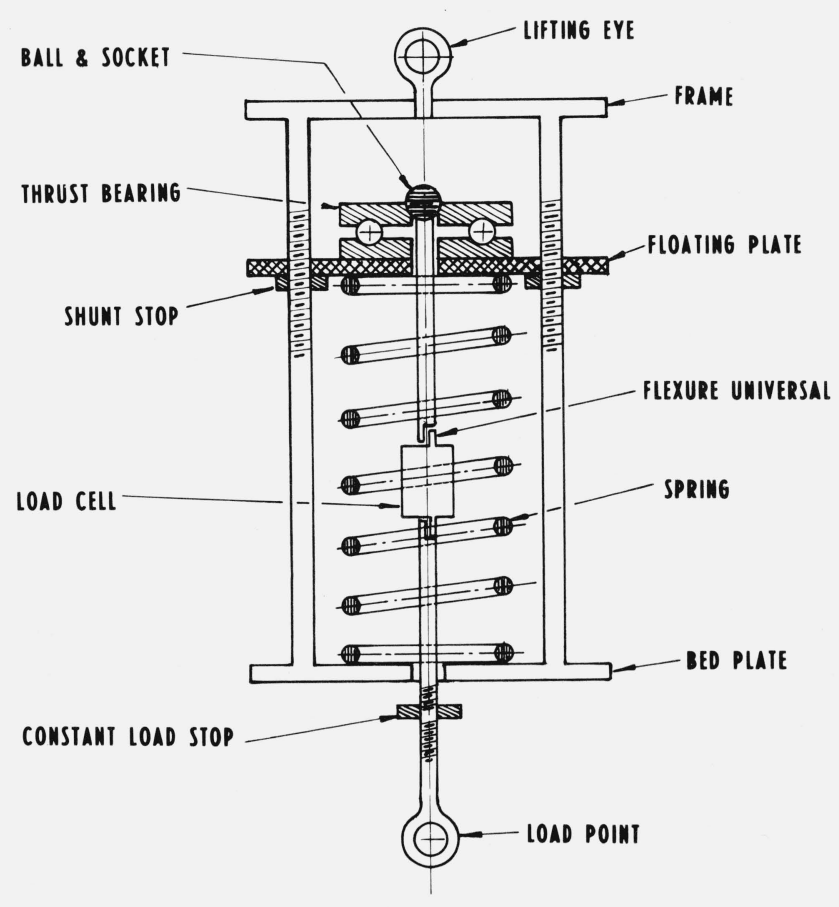

FigURE 1. Schematic of the load cell comparator.

Initially a force is applied to the load point and acts on the load cell and floating plate, thereby causing the spring to compress. The shunt stops are adjusted to halt spring compression at 90 percent full load, thus providing a stiff support. At 100 percent load, the constant load stop is adjusted to prevent more than 10 percent removal when loads to be compared are exchanged.

motion of the floating plate is restricted by vertical rods that are part of the frame. The rods do not, however, impede vertical motion of the spring and plate.

Resting on top of the floating plate are a thrust bearing and ball-and-socket assembly. The ball-and-socket permits gross vertical misalignment of the cell and frame during initial assembly. Whereas the bearing allows the spring to rotate during compression and extension, it also removes torque from the cell during the loading cycle as well.

Adjustable shunt stops are set to halt vertical motion of the floating plate just before full loading is reached, thus providing a rigid suspension of the cell. When the load is removed, slight vertical motion of the spring and cell is permitted before reaching the constant load stop. This stop is adjusted to provide about 90 percent of full load to the cell when the load is removed.

Universal flexure joints provide a repeatable load axis within their range of articulation. Otherwise, minor mechanical misalignment that occurs during the load/unload cycle would degrade cell performance.

In addition to the above components, a hydraulic lifting mechanism is required as well as a weight transport system. Dollies and tracks provide excellent weight handling up to $1000 \mathrm{~kg}$ for this device. Also, an electronic device is required to indicate the load cell output signal. Figure 2 shows schematically the complete weighing system.

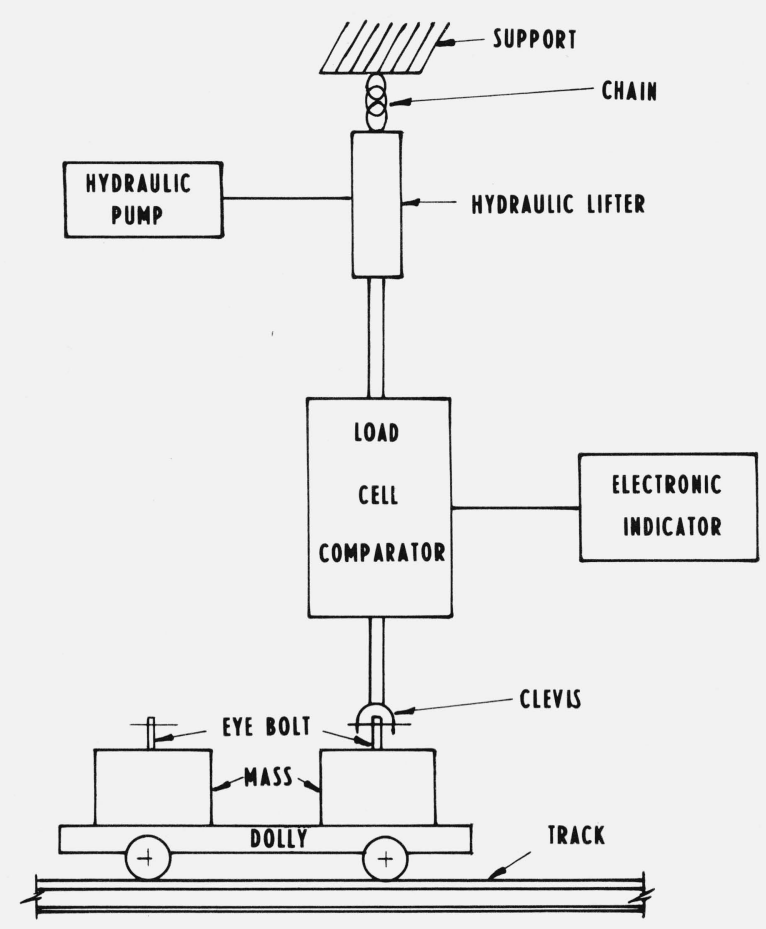

FIGURE 2. The complete load cell weighing system.

The hydraulic lifter provides a means to load and unload the comparator, whereas the dolly and track are the transport system for exchanging loads.

\section{Components}

The components shown in figure 1 are "off-the-shelf" except for the frame and the ball-and-socket assemblies. These assemblies are easily fabricated; the important criterion is structural strength. However, to increase utility, the shunt and load stops should be adjustable over a wide range of loading.

\subsection{Load Cell}

A commercially-available load cell with solid state strain gages was selected for use in the comparator. The following pertinent specifications are listed as given by the manufacturer: (a) sensitivity, $30 \mathrm{mv} / \mathrm{V}$ open circuit at capacity load; (b) excitation voltage, $5 \mathrm{~V}$ dc or ac rms; (c) capacity, $900 \mathrm{~kg}$; (d) deflection; full scale, $0.05 \mathrm{~mm}$; (e) non-linearity, \pm 0.1 percent full scale; (f) hysteresis, \pm 0.5 percent full scale; (g) repeatability, \pm 0.05 percent full scale; (h) side load error, 0.1 percent full scale per $1^{\circ}$ off axis; (i) thermal effect on zero, less than 0.0025 percent $/{ }^{\circ} \mathrm{C}$.

\subsection{Flexure Universal}

Two flexure universal joints are required, one above and one below the load cell. Flexure stiffness varies with capacity 
and should be matched to the expected load for best performance. For the device presented here, commercial units with a $450 \mathrm{~kg}$ load capacity were used.

\subsection{The Spring}

In choosing the spring, practical considerations are important. For example, a very stiff spring with little deflection when loaded would be impractical as the load and shunt stop adjustment would be critical. These adjustments would then entail accurately machined parts and cause difficulty in making nominal load changes. A spring which is too short limits the load range of the comparator, impairing its usefulness.

For simplicity of design, an open coil compression spring about $50 \mathrm{~cm}$ in length which will compress about $1 \mathrm{~cm}$ when loaded with a $20 \mathrm{~kg}$ weight was chosen. Although less important, the use of a spring with a $15 \mathrm{~cm}$ outside diameter and having flat ends further simplified construction. A few such springs from a local automobile junk yard were found to be suitable. The test consisted of supporting the author's weight on the spring and measuring the displacement with a meter stick.

\subsection{Thrust Bearing}

The only considerations given the thrust bearing were that it be a precision, low-friction type with sufficient static-load capacity. There is virtually no dynamic loading of the bearing.

\subsection{Electronic Indicator}

The indicator chosen should not degrade the overall performance of the comparator. The goal here was a reproducibility of $0.00025 \mathrm{~kg}$ for a load of $250 \mathrm{~kg}$ or about $1 \mathrm{ppm}$ of the applied load. Therefore the indicator must be able to reproducibly indicate the small differences in cell output.

On hand was a current bridge of commercial origin designed for indicating load cell output with a resolution of $0.5 \mathrm{ppm}$ of the loads involved. The linearity and reproducibility of the bridge were adequate for its use as a comparator. In conjunction with the bridge, an external $5 \mathrm{~V}$ dc power supply and electronic null detector for indicating bridge balance were required. In addition, the detector's internal amplifier was used to drive a high-impedance strip chart recorder for recording some of the observations.

\section{The Complete Weighing System}

To make full use of the mass comparator, some attention must be paid to its support and loading. A stiff supporting structure for the comparator is an obvious requirement. Slow, uniform loading is accomplished with a hydraulic lifter (see fig. 2). Mechanical shock from improper loading is known to degrade load cell performance. Also, indexing the position of the object to be loaded with respect to the load point results in a reproducible load position and minimizes loading error.

\section{Method of Test}

As previously mentioned, the difference measurement between two nominally equivalent objects is basic to high precision mass metrology. Usually, a combination of differences between several such objects is observed and the difference equations are solved for the unknown value of mass. This statistical treatment is often referred to as a "weighing design" or "series" [2].

With this weighing design in mind, two weights of equal density are used to simulate the six possible combinations of four weights. The use of only two artifacts in a four weight design permits the influence of time, temperature, loading effects, etc. on the instruments to be determined, with significant operational simplification. In addition, buoyant forces on the weights are almost equal and can be neglected. Because zero-drift is usually present, a time sequence of $45 \mathrm{~s}$ was maintained for exchanging loads and a delay of 30 $\mathrm{s}$ after loading before recording data was maintained. However, the loading time for a small weight of mass $\Delta$ was insignificant and no applicable time was allotted. The purpose of $\Delta$ is discussed later in the text.

To demonstrate load cell performance without constant loading, three weighing series were performed from an Ibeam support. Likewise, three more series with constant loading were performed from the same support. Experience gained in collecting the above data suggested that a strip chart recorder would provide beneficial time integration, offnull operation, and operator convenience. Therefore, two more series were made with strip chart recording of observations. Finally the comparator was suspended from a chain hoist supported by an A-frame for an additional three series. The I-beam and A-frames represent typical support methods.

The success of these tests led to a noteworthy application of the comparator that demonstrates its versatility, that is, gravimetric calibration [3] of a $378 \mathrm{~L}$ (100 gal) test measure, a device itself used in volumetric calibrations of large tanks. This gravimetric calibration was accomplished by weighing a test measure first empty and then filled with water. From these data (and the density of the water) the internal volume of the measure can be calculated. Several such calibrations were performed. The mass of the test measure was approximately $99 \mathrm{~kg}$ empty and $477 \mathrm{~kg}$ when filled with water.

\subsection{Data Reduction}

The process of loading weight $\mathrm{A}$ on a comparator, observing the response and then substituting weight $\mathrm{B}$ in place of $\mathrm{A}$ and again noting the response, is called a substitution weighing. A more useful modification of the above descrip- 
tion is called a "double substitution" and is represented by the following observational format:

\begin{tabular}{|c|c|c|}
\hline Observation & & Load \\
\hline $\mathrm{O}_{1}$ & $\propto$ & Mass $A$ \\
\hline $\mathrm{O}_{2}$ & $\propto$ & Mass $B$ \\
\hline $\mathrm{O}_{3}$ & $\propto$ & Mass $B+\Delta$ \\
\hline $\mathrm{O}_{4}$ & $\propto$ & Mass $A+\Delta$ \\
\hline
\end{tabular}

where $\Delta$ is the small weight of known mass, known as the "sensitivity weight", chosen to be larger than the difference $A-B$ and is used to calibrate the bridge output in units of mass. For this test $\Delta$ was $22.68 \mathrm{~g}(0.0500 \mathrm{lb})$.

These four observations are conventionally reduced to yield a single difference value as follows:

$$
A-B=\left(\frac{\mathrm{O}_{1}-\mathrm{O}_{2}+\mathrm{O}_{4}-\mathrm{O}_{3}}{2}\right)\left(\frac{\Delta}{\mathrm{O}_{3}-\mathrm{O}_{2}}\right)
$$

The above observation format was used to observe the six mass differences of each simulated series. Advantages of the double substitution are well known and are not discussed here.

\section{Data}

Each weighing series yields six values for $A-B$ as shown in table 1 . The mean value, $\bar{\chi}$, the estimated standard deviation, $S_{\chi}$, and the estimate of the standard deviation of the mean, $S_{\bar{\chi}}$, are given for each series.

As was expected, the data of table 1 indicate significant improvement in precision when the load cell is used with, rather than without, constant loading. This limited test shows that an improvement of at least one order in magnitude is attained. Furthermore, considering only the constant loading data, the 90 percent confidence interval of any mean value overlaps that of any other value, except in one instance, thus demonstrating the comparator is yielding consistent results. Figure 3 demonstrates this fact graphically. The last three values of figure 3 appear to indicate a trend. The observed slope may be fortuitous, a function of the apparatus, or most likely, an indication of apparent changes in mass. With the high level of precision available, such apparent mass changes can be attributed to the weight volumes or temperatures not being equal.

As mentioned previously, the comparator was used successfully to determine the capacity of a $378 \mathrm{~L}(100 \mathrm{gal})$ test measure; table 2 summarizes these results. Intrinsically, reproducibility of the test measure capacity determination, about $20 \mathrm{ppm}$, is independent of instrumentation. That is, errors in reading the meniscus, variability of entrained gases, etc., limit the reproducibility of the water filling ind hence the calculated capacity.

\section{Conclusion}

The data reported here attest to the benefit of constant loading of load cells and the ease and practicality of doing so

TABLE 1

Each set of data shown is the repeated measured difference in mass (A-B), expressed in grams, of two $225 \mathrm{~kg}(500 \mathrm{lbs}$.) test weights.

\begin{tabular}{r|c|c}
\hline \hline I-beam support & without constant loading & without integration \\
\hline A-B & A-B & A-B \\
21.86 & 14.60 & 6.03 \\
16.87 & 26.31 & 1.59 \\
14.52 & 23.59 & 26.40 \\
15.29 & 14.47 & 17.46 \\
22.59 & 30.48 & 1.72 \\
13.83 & 35.24 & 14.70 \\
$\bar{\chi}=17.49$ & 24.12 & 11.32 \\
$S_{\chi}=1.81$ & 8.40 & 9.91 \\
$S_{\bar{\chi}}=1.56$ & 3.43 & 4.05 \\
I-beam support & with constant loading & without integration \\
\hline A-B & A-B & A-B \\
18.14 & & \\
17.60 & 16.42 & 17.87 \\
17.55 & 15.88 & 18.10 \\
16.69 & 15.88 & 16.69 \\
16.60 & 17.46 & 17.01 \\
15.97 & 17.83 & 16.06 \\
$\bar{\chi}=17.09$ & 16.24 & 20.23 \\
$S_{\chi}=$ & 16.62 & 17.66 \\
$S_{\bar{\chi}}=$ & 0.83 & 0.60 \\
0.33 & 0.34 &
\end{tabular}

\begin{tabular}{|c|c|c|}
\hline I-beam support & with constant loading & with integration \\
\hline$A-B$ & $A-B$ & \\
\hline 17.01 & 15.83 & \\
\hline 15.88 & 16.51 & \\
\hline 15.74 & 16.28 & \\
\hline 17.60 & 16.37 & . \\
\hline 16.92 & 15.88 & \\
\hline 16.65 & 16.60 & \\
\hline $\bar{\chi}=16.62$ & 16.24 & \\
\hline$S_{\chi}=0.71$ & 0.32 & \\
\hline$S_{\bar{\chi}}=0.29$ & 0.13 & \\
\hline A-frame support & with constant loading & with integration \\
\hline$A-B$ & $A-B$ & $A-B$ \\
\hline 16.46 & 16.51 & 16.51 \\
\hline 15.60 & 16.69 & 17.24 \\
\hline 16.42 & 16.33 & 16.96 \\
\hline 16.78 & 17.10 & 16.78 \\
\hline 16.33 & 16.69 & 17.01 \\
\hline 16.78 & 16.96 & 17.55 \\
\hline $\bar{\chi}=16.40$ & 16.71 & 17.01 \\
\hline$S_{\chi}=0.43$ & 0.28 & 0.36 \\
\hline$S_{\bar{\chi}}=0.18$ & 0.12 & 0.15 \\
\hline
\end{tabular}


with a simple spring. The load cell comparator can have a precision of $3 \mathrm{ppm}$, can be very portable with a large range of capacity, and can make mass comparisons of large, unwieldy objects.

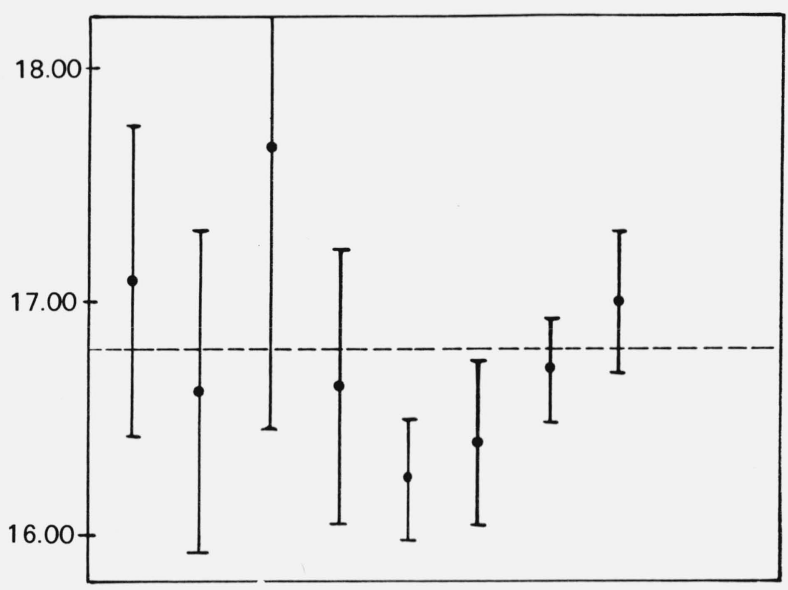

Figure 3. Points shown are the mean values, $\bar{\chi}$, for the difference between two weights $(A-B)$ in grams for each simulated series with constant loading.

Bars are the associated 90 percent confidence interval and the dashed line is the average of all plotted values.

TABLE 2

Capacity values assigned by load cell weighing to a $378 \mathrm{~L}$ (100 gal) test measure.

378.587 liters

378.579

378.571

378.583

378.568

$\bar{\chi}=378.578$

$S_{\bar{\chi}}=0.004$

\section{Discussion}

Other possible spring arrangements may be of advantage. For instance, a group of parallel springs in tension may simplify initial assembly. The removal or addition of springs could increase or decrease the comparator capacity by an order of magnitude. Changing capacity, would, of course, require changing the load cell and flexures as well. Commerically available hydraulic springs based on the compressibility of a fluid offer the designer many possibilities for improvement as well as reduction in size.

Additionally, the application of modern ac bridge techniques tailored to the comparator may have advantages for observing load cell output.

Lastly, in the author's opinion, it would be very useful to many laboratories if a $30 \mathrm{~kg}$ comparator could be built with a precision of $10 \mathrm{ppm}$ or better. A successful device would offer speed, ruggedness and precision not found elsewhere.

The author wishes to express special thanks to Horace A. Bowman, his mentor in this endeavor and the mentor of many others until his recent retirement. The enthusiastic consultation of John F. Houser and his assistance with the test measure calibration are gratefully acknowledged. Also thanks are due to Paul E. Pontius, Chief of the former Mass and Volume Section, for supporting this work.

\section{References}

[1] Bowman, H. A. and Almer, H. E., Minimization of the arrestment error in one-pan, two-knife balance systems, J. Res. Nat. Bur. Stand. 67C, (Eng. and Instr.), No. 3, 227-235 (July-Sept. 1963).

[2] Cameron, J. M., Croarkin, M. C. and Raybold, R. C., Designs for the Calibration of Standards of Mass, Nat. Bur. Stand. (U.S.) Tech. Note 952, 64 pages (June 1977).

[3] Schoonover, R. M. and Houser, J. F., Uncertainties in the calibration of large vessels, National Bureau of Standards Report 10081. 